\title{
Ultrasonic Bonding of Membrane-Electrode-Assemblies of Fuel Cells
}

\author{
Dung-An Wang \\ Graduate Institute of Precision Engineering, National Chung Hsing University, 240 Kuo Kuang Road, Taichung, 40227, Taiwan, ROC \\ E-mail:daw@nchu.edu.tw
}

\begin{abstract}
Ultrasonic bonding has a great potential for manufacturing of membrane electrode assemblies (MEAs) of fuel cells (FCs) due to its short process cycle time and low energy consumption. Before introduction of the bonding process into the industry, a detailed and elaborate investigation of the effects of the processing parameters on the bonding quality is necessary. We develop a finite element model of the ultrasonic bonding for MEAs of FCs. The model can be used as a computational framework for initial evaluation of the effectiveness of ultrasonic boding for MEAs of FCs.
\end{abstract}

Keywords - Ultrasonic bonding; membrane electrode assembly; FC

\section{INTRODUCTION}

As production volume and quality standard of FCs increase, manufacturers in the industry must adopt automated processes to meet market demands. Continuous roll-to-roll process is a key method for rapid and efficient upscale manufacturing of MEAs of FCs. Examples could be spray coating of an electrode active layer onto a substrate membrane, or tape casting of an anode support. 3M has implemented a roll-to-roll process for continuous production of MEAs. In that process, traditional hot pressing for bonding MEA components was replaced by a hot rolling step. Ultrasonic bonding is an alternative method for production of MEAs due to its short process cycle time and low energy consumption. It is capable of creating a solidstate bond between dissimilar materials and requires no melting of the components and no adhesives. Beck et al. [1] investigated ultrasonic bonding of MEAs for low temperature proton exchange membrane FCs (PEMFCs). They demonstrated that the process cycle time and energy consumption of the ultrasonic bonding were nearly two orders-of-magnitude less than the thermal bonding.

In this work, we presented a thermal-mechanical analysis of roll-to-roll ultrasonic bonding of MEAs of FCs. The joining of gaskets to both sides of a polymer electrolyte membrane by ultrasonic bonding was simulated by a simplified three-dimensional configuration. Temperature and plastic strain distributions were examined. Friction work was evaluated. The finite element model can serve as a platform for numerical investigation of the effects of the processing parameters on quality of ultrasonic bonding of MEAs of FCs.

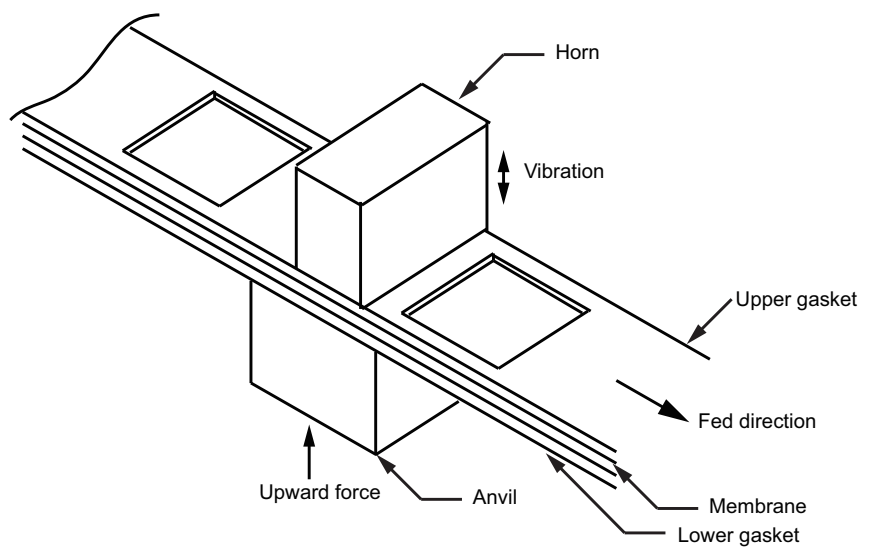

Fig. 1 a schematic of a roll-to-roll ultrasonic bonding setup for MEAs of FCs

\section{ULTRASONIC BONDING OF MEAS}

Fig. 1 of a schematic of a roll-to-roll ultrasonic bonding setup for MEAs of FCs, where two gaskets are bonded to the upper and lower surface of a PEM, respectively, using ultrasonic bonding. The gaskets and the membrane are fed into an ultrasonic horn which provides ultrasonic vibration for bonding, and they are clamped onto an anvil. The vibration direction of the horn is indicated by an arrow. The arrow at the fore end of the membrane indicates the fed direction of the MEAs in a roll-to-roll process. An upward force is applied at the MEAs by the anvil. After the gaskets are bonded to both sides of the membrane, an electrode can be spray coated on the membrane through the openings of 
the gaskets. The process may prevent damage to the MEAs by ultrasonic bonding, as reported by Lee [2].
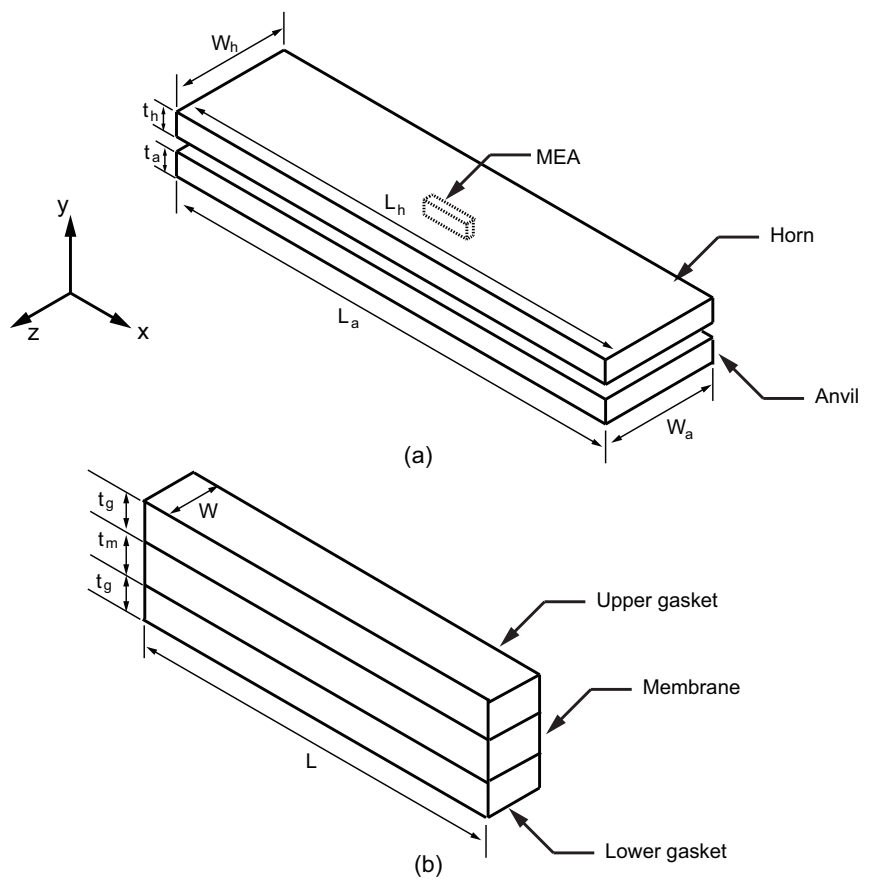

Fig. 2 a schematic of a roll-to-roll ultrasonic bonding setup for MEAs of FCs

The dimensions of the MEAs, the horn and the anvil considered in this investigation are shown in Fig. 2. A Cartesian coordinate system is also shown in the figure. As shown in Fig. 2(a), the horn and the anvil have thicknesses $t_{h}, t_{a}$ of $1 \mathrm{~mm}$, widths $W_{h}, W_{a}$ of $5 \mathrm{~mm}$, and lengths $L_{h}$, $L_{a}$ of $20 \mathrm{~mm}$. The thicknesses of the membrane and the gaskets are $t_{m}(=0.2 \mathrm{~mm})$ and $t_{g}(=300 \mu \mathrm{m})$, respectively, see Fig. 2(b). The MEA has a width $W$ of $0.3 \mathrm{~mm}$, and a length $L$ of $2 \mathrm{~mm}$. A sinusoidal displacement $u$ of the horn is given as

$$
u(t)=a \sin (2 \pi f t)
$$

where $a$ and $f$ represent amplitude and frequency of the vibration of the horn, respectively, and $t$ is the time.

The ultrasonic process under investigation is applied to join three thin sheets of dissimilar materials. An ultrasonic horn with a probe tip plunges into the upper gasket and a backing tool, the anvil, beneath the lower gasket applies the upward force. The upward force and the vibration displacement are maintained for an appropriate time to generate frictional heat. Then, heated and softened material adjacent to the ultrasonic tip deforms, a solid state bond is made between the surfaces of the gaskets and the membrane. For the ultrasonic process, the important processing parameters are the tool geometry, the vibration amplitude $a$ and the frequency $f$, the cycles of ultrasonic bonding (holding time) and the upward force (bonding pressure). During the process in this investigation, the upward force is kept constant. Initially, the upward force increases almost linearly for a period of time. Then the upward force is kept nearly constant during the ultrasonic bonding process. After the pressure reaches the desired level, the ultrasonic tip contacts to the top surface of the upper gasket. For the case of bonding silicone rubber gaskets to Nafion membrane, the temperature at the interfaces between the contact surfaces of the tip, the MEA and the anvil should be kept higher than the melting point of the membrane and lower than that of the gasket. This bonding strategy was reported by Uchida and Ashibe [3].

\section{FINITE ELEMENT ANALYSIS}

A three-dimensional finite element model is considered to examine the effects of processing parameters on the stress/strain and temperature distributions of the MEAs. As shown in Fig. 2, a velocity $V$ is applied in the $x$ direction to the gaskets and the membrane to represent the roll-to-roll manufacturing process. The horn and the anvil are modelled as rigid elements. The displacement in the $y$ direction of the horn follows the sinusoidal displacement function as Equation (1). The $y$ displacement of the anvil is free for application of a constant pressure during ultrasonic bonding. The $x$ displacements of the anvil and the horn are constrained. The vibration amplitude and frequency, constant pressure, and holding time $t_{f}-t_{i}$ for the ultrasonic process are $5 \mu \mathrm{m}, 20 \mathrm{kHz}, 0.3 \mathrm{MPa}$, and $0.5 \mathrm{msec}$, respectively. It is assumed that the heat generated at the interfaces of the model due to frictional sliding is all converted into dissipated energy and distributed evenly between the two interacting surfaces. No heat transfer between the rigid bodies and the gaskets is also assumed.

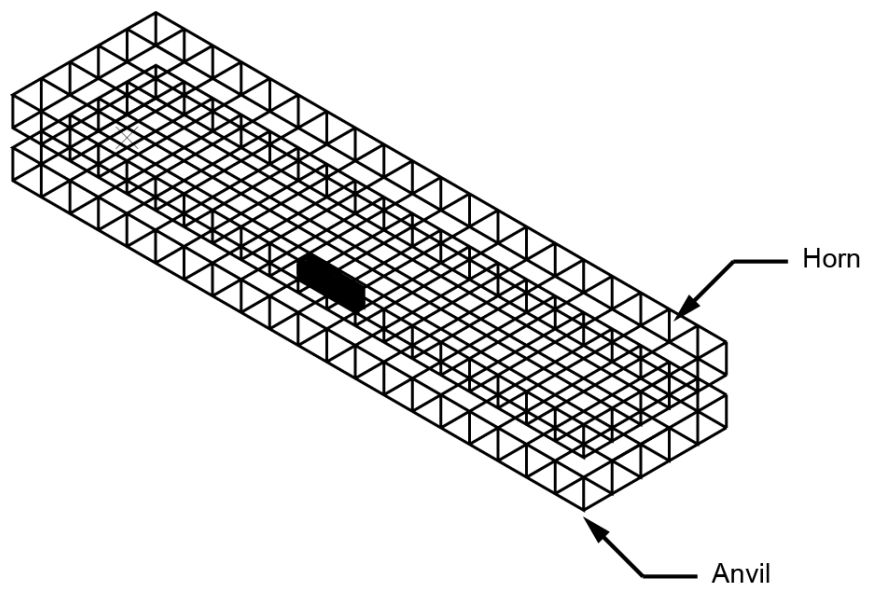

Fig. 3 A mesh for a finite element model

Fig. 3 shows a mesh for a finite element model. The wavelengths of the wave in a typical silicone rubber and Nafion membrane given a frequency of $20 \mathrm{kHz}$ are nearly 50 $\mathrm{mm}$ and $200 \mathrm{~mm}$, respectively. Therefore, the wave propagation phenomena can be neglected in this investigation. Note that a mesh design with 10 elements per wavelength is suggested to resolve a wave pattern well [4]. Fig. 4(a) is a front view of the mesh. Fig. 4(b) shows a close-up view of the mesh of the MEA. The finite element model has 560 8-node trilinear displacement and temperature, reduced integration with hourglass control. 


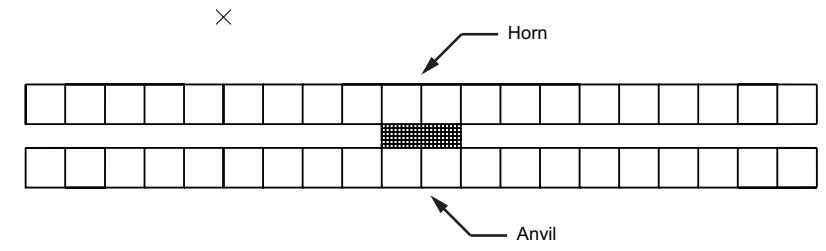

(a)

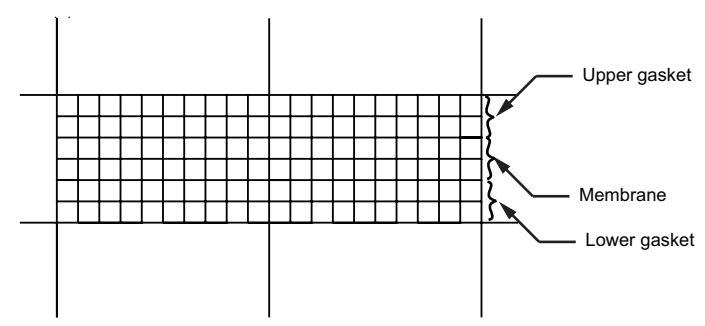

(b)

Fig. 4 (a) A front view of the mesh, (b) a close-up view of the mesh of the MEA

In this investigation, the materials of the gasket and the membrane are taken as a silicone rubber and the DuPont Nafion 112 (perfluorosulfonic acid), respectively. Steel is the material used for the horn and the anvil. The physical properties of a silicone rubber, Nafion 112 [5] and a steel are listed in Table 1.

TABLE I

PHYSICAL PROPERTIES OF THE SILICONE RUBBER AND NAFION 112.

\begin{tabular}{|l|l|l|l|}
\hline & $\begin{array}{l}\text { Silicone } \\
\text { rubber }\end{array}$ & Nafion 112 & Steel \\
\hline $\left.\begin{array}{l}\text { Thermal conductivity } \\
k(\mathrm{~W} \mathrm{~m} \mathrm{~K}\end{array}\right)$ & 0.200 & 0.259 & 43 \\
\hline Density $\rho\left(\mathrm{kg} \mathrm{m}^{-3}\right)$ & 1070 & 2000 & 7800 \\
\hline Poisson's ratio $v$ & 0.5 & 0.25 & 0.3 \\
\hline $\begin{array}{l}\text { Coefficient of thermal } \\
\text { expansion } \alpha\left(\mathrm{K}^{-1}\right)\end{array}$ & $342 \times 10^{-6}$ & $123 \times 10^{-6}$ & $12 \times 10^{-6}$ \\
\hline $\begin{array}{l}\text { Heat capacitance } \\
C_{p}\left(\mathrm{~J} \mathrm{~kg}^{-1} \mathrm{~K}^{-1}\right)\end{array}$ & 1465 & 1050 & 460 \\
\hline
\end{tabular}

The silicone rubber is assumed to be a hyperelastic material. The Ogden material model is used to describe the non-linear stress-strain behaviour of the rubber. The shear modulus, the strain hardening coefficient and the initial bulk modulus of the Ogden model are taken as $12.605 \mathrm{kPa}, 4.320$ and $2 \mathrm{MPa}$, respectively, for a silicone rubber [6]. The membrane is modelled as a linear-elastic, plastic material with isotropic hardening. The mechanical properties of the membrane within a temperature range from 25 to $85{ }^{\circ} \mathrm{C}$ at a relative humidity of $30 \%$ are listed in Table 2 [7]. No swelling of the Nafion 112 membrane is assumed in the presence of moisture. The Young's modulus and the yield strength of the steel are taken as $200 \mathrm{GPa}$ and $250 \mathrm{MPa}$.
TABLE II

MECHANICAL PROPERTIES OF THE MEMBRANE NAFION 112 [7].

\begin{tabular}{|l|l|l|l|l|}
\hline & \multirow{2}{*}{$\begin{array}{l}\text { Young's } \\
\text { modulus (MPa) }\end{array}$} & \multicolumn{3}{|c|}{ Yield strength (MPa) } \\
\cline { 3 - 5 } & $\varepsilon^{p l}=0$ & $\varepsilon^{p l}=0.05$ & $\varepsilon^{p l}=0.25$ \\
\hline $25{ }^{\circ} \mathrm{C}$ & 197 & 6.76 & 7.16 & 9.71 \\
\hline $45{ }^{\circ} \mathrm{C}$ & 161 & 5.67 & 5.70 & 7.31 \\
\hline $65{ }^{\circ} \mathrm{C}$ & 148 & 5.14 & 5.30 & 6.55 \\
\hline $85{ }^{\circ} \mathrm{C}$ & 121 & 3.61 & 4.16 & 5.04 \\
\hline
\end{tabular}

The commercial finite element program ABAQUS is employed to perform the computations. Zero stress state is assumed at the beginning of the analyses. The initial temperature is set as $25{ }^{\circ} \mathrm{C}$. An initial velocity $V(=4 \mathrm{~m} / \mathrm{sec})$ is applied in the $x$ direction to the gaskets and the membrane for the roll-to-roll process. The value of the friction coefficient at the interfaces between all contact pairs is selected as 0.3 . In order to introduce conductive heat transfer between closely adjacent (or contacting) surfaces, the thermal gap conductance model in Abaqus is used and the value of the gap conductance as a function of the gap clearance in an atmosphere environment is listed in Table 3. These values are estimated based on a model proposed by Song et al. [8].

TABLE III

GAP CONDUCTANCE AS A FUNCTION OF THE GAP CLEARANCE.

\begin{tabular}{|l|l|}
\hline $\begin{array}{l}\text { Gap conductance } \\
\left(\mathrm{MJ} \mathrm{sec}^{-1} \mathrm{~m}^{-1} \mathrm{~K}^{-1}\right)\end{array}$ & $\begin{array}{l}\text { Gap clearance } \\
(\mathrm{mm})\end{array}$ \\
\hline $2.57 \times 10^{-3}$ & $1 \times 10^{-5}$ \\
\hline $2.57 \times 10^{-4}$ & $1 \times 10^{-4}$ \\
\hline $8.57 \times 10^{-5}$ & $3 \times 10^{-4}$ \\
\hline $5.14 \times 10^{-5}$ & $5 \times 10^{-4}$ \\
\hline $3.67 \times 10^{-5}$ & $7 \times 10^{-4}$ \\
\hline $2.57 \times 10^{-5}$ & $1 \times 10^{-3}$ \\
\hline
\end{tabular}

Abaqus/Explicit is chosen to solve the transient thermal and structural problem. The transient solution is obtained by marching forward through time in small time increments. Explicit methods are conditionally stable. A maximum time step in the dynamic analysis using explicit methods should be less than the shortest time for a wave to cross any element in the mesh, $2.5 \times 10^{-6} \mathrm{sec}$. The shortest time can be taken as the minimum element size divided by the wave speed. As suggested by Abboud et al. [4], a time step should be smaller than one-tenth of the period of the highest frequency of interest in wave phenomena in order to obtain desired resolution and accuracy. ABAQUS/Explicit automatically adjusts the stable time increment during the analysis. In this investigation, we simulate the initial 10 cycles of the process. 


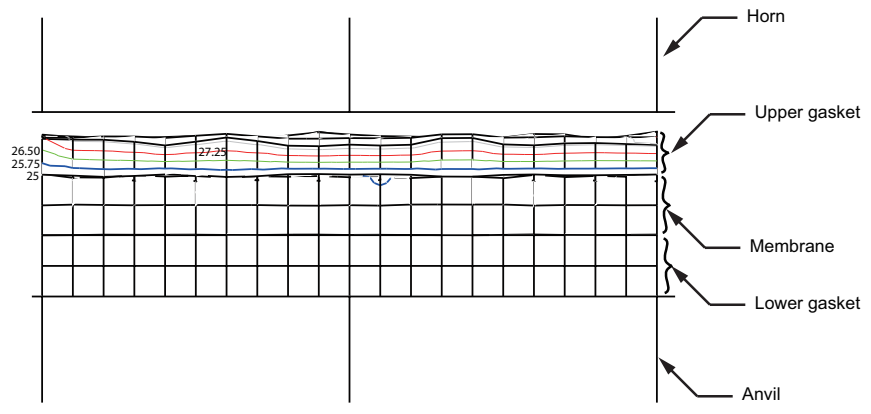

Fig. 5 Temperature distribution near the horn after 10 cycles of the process

\section{RESULTS}

Temperature distribution near the horn at the end of the simulation is shown in Fig. 5. Temperatures in the upper gasket range from $25{ }^{\circ} \mathrm{C}$ to $28{ }^{\circ} \mathrm{C}$. The temperature near the interfaces between the gaskets and the membrane is nearly $25{ }^{\circ} \mathrm{C}$. The highest temperature achieved in this investigation is much lower than the melting point of the Nafion, near $230{ }^{\circ} \mathrm{C}$ [9]. The cycles of ultrasonic bonding and the bonding pressure may be increased to obtain higher temperatures at the interfaces between the gaskets and the membrane to facilitate boning.

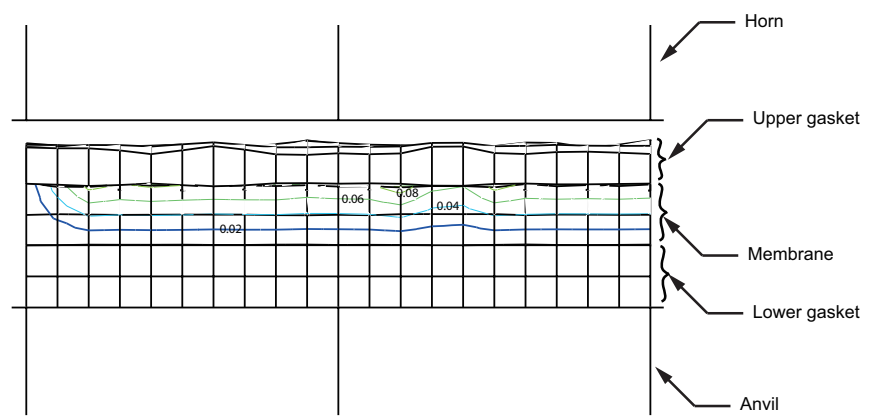

Fig. 6 Temperature distribution near the horn after 10 cycles of the process

Fig. 6 shows the contours of the equivalent plastic strain near the horn. For the selected processing parameters, the plastic strain is relatively small, and the highest equivalent plastic strain is only 0.14 . The equivalent plastic strain near the interface between the higher gasket and the membrane is much higher than between the lower gasket and the membrane. Note that there is no plastic strain in the gaskets since they are modelled as hyperelastic material. For effective ultrasonic bonding, severe plastic deformation may be required at the interfaces for bonding. Further investigations of the effects of processing parameters on the induced plastic strain are needed in order to achieve strong bonding.

\section{CONCLUSIONS}

A finite element model for ultrasonic bonding of MEAs of FCs is developed. Investigations of the processing parameters, such as cycles of ultrasonic bonding, bonding pressure, frequency of the ultrasonic bonding can be carried out using the model. Some initial studies of induced temperature and equivalent plastic strain distributions are conducted to demonstrate the effectiveness of the finite element model.

\section{ACKNOWLEDGMENT}

We would like to thank National Center for HighPerformance Computing (NCHC), Taiwan for permits to use the Abaqus software.

\section{REFERENCES}

[1] J. Beck, D. Walczyk, C. Hoffman and S. Buelte, "Ultrasonic bonding of membrane electrode assemblies for low temperature proton exchange membrane FCs," Journal of FC Science and Technology, vol. 9, 051005, Aug. 2012.

[2] H.-H. Lee, Method for Manufacturing FC Membrane-Electrode Assembly Ultrasonic Vibration Bonding, United States Patent Application Publication, Pub. No.: US 2013/0068371 A1, Mar. 21, 2013.

[3] Y. Uchida and M. Ashibe, "Rubber/Resin Ultrasonic Bonding Method.," U.S Patent 7699950 B2, April 20. 2010.

[4] N. N. Abboud, G. L. Wojcik, D. K. Vaughan, J. Mould, D. J. Powell and L. Nikodym, "Finite element modeling for ultrasonic transducers," Proc. SPIE 3341, Medical Imaging 1998: Ultrasonic Transducer Engineering, 19 (May 1, 1998); doi:10.1117/12.308015.

[5] DuPont, Product Information: Nafion PFSA Membranes N-112, NE1135, N-115, N-117, NE-1110 Perfluorosulfonic Acid Polymer, NAE101, 2004.

[6] J. L. Sparks, N. A. Vavalle, K. E. Kasting, B. Long, M. L. Tanaka, P. A. Sanger, K. Schnell, and T. A. Conner-Kerr, "Use of silicone materials to simulate tissue biomechanics as related deep tissue injury," Advances in Skin \& Wound Care, vol. 28, pp. 59-68, February 2015.

[7] A. Kusoglu, A. M. Karlsson, M. H. Santare, S. Cleghorn, and W. B. Johnson, "Mechanical behavior of FC membranes under humidity cycles and effect of swelling anisotropy on the fatigue stresses," Journal of Power Sources, vol. 170, pp. 345-358, 2007.

[8] S. Song, M. M. Yovanovich, and F. O. Goodman, "Thermal gap conductance of conforming surfaces in contact," Journal of Heat Transfer, vol. 115, pp. 533-540.

[9] Y. Kawano, Y. Wang, R. A. Palmer, and S. R. Aubuchon, "Stressstrain curves of Nafion membranes in acid and salt forms," Polímeros: Ciência e Tecnologia, vol. 12, n 2, pp. 96-101, 2002. 